\title{
Rezension zu: Volk, Theresia (2019): Spielen, um zu gewinnen. Macht und Wirksamkeit in Organisationen.
}

\author{
Göttingen: Vandenhoeck \& Ruprecht. 104 Seiten, 15,00€
}

\author{
Martin Winand ${ }^{1,2}$ \\ Angenommen: 2. Januar 2021 / Online publiziert: 26. Januar 2021 \\ (c) Der/die Autor(en) 2021
}

„Es geht immer nur um Macht.“ Theresia Volk fordert dazu auf, im beruflichen Kontext nicht nur Machtkompetenz $\mathrm{zu}$ entwickeln, sondern darüber hinaus auch ein wenig Spielfreude, wenn es darum geht, sich und seine Ideen gegen Konkurrenz durchzusetzen. Hierbei dominieren Interessen, Bedürfnisse und Ängste von Menschen über Rationalität. Gleich mehrfach arbeitet Theresia Volk hierbei sich aktuell vollziehende Paradigmenwechsel heraus. Letztlich werden die traditionellen Legitimationsprinzipien, Wahrheit und Konsens, durch Chance und Option ins Wanken gebracht. Nach Theresia Volk fehlt es an der Vermittlung von Machtkompetenz, die über Erfahrung erworben wird. Dies macht sie zu einer Aufgabe für Beratende. Hervorbringen, Sichtbarmachen und Aufbrechen von Machtprozessen sowie das Entwickeln von Machtkompetenzen wird zur Essenz einer machtbewussten Beratung und ein machtpolitisch abstinentes Selbstverständnis von Beratenden wird so ad absurdum geführt. Wenngleich normative und ethische Aspekte von Macht sehr im Hintergrund bleiben und nur vereinzelt anklingen, wirft Theresia Volk doch systematisch und schlüssig zahlreiche relevante Fragen auf und inspiriert den Leser zu kritischer Selbstreflexion und Positionierung. Bis zur Schlussaussage - ,Wir dürfen danach streben, zu gewinnen. Wir dürfen Lust auf Macht haben“ liegen 90 Seiten vor dem Leser, in denen Theresia Volk das Phänomen Macht und Wirksamkeit aus unterschiedlichen Blickwinkeln erörtert.

Theresia Volk steigt ohne langen Anlauf in ihr Thema Macht ein. Macht ist dabei für sie vor allem ein Bezie-

\section{Martin Winand}

martin.winand@focuslotsen.de

1 Bonn, Deutschland

2 Universität Kassel, Kassel, Deutschland hungsphänomen. Zunächst fällt es schwer, die Struktur in ihren Ausführungen zu erkennen. In einer ebenso sinnvollen wie kurzen Literatursichtung beleuchtet sie sowohl Macht aus individueller und organisationaler Perspektive. Machtaspekte neuer Arbeitswelten runden diese Betrachtungen ab. Vollständig erschließt sich die gewählte Struktur erst gegen Ende des Buches, wenn alle die sorgfältig vorbereiteten Elemente im Konzept einer machtbewussten Beratung zusammengefügt werden.

Als zentralen Einflussgrößen werden hierbei Ego und Alter als gegensätzliche Machtperspektiven herausgearbeitet. Diese äußern sich nach Theresia Volks Darstellung zu Allianzen oder Konfrontationen und formen Systeme wiederstreitende individuellen Interesse. Spielregeln, Strategie und Handlungen, werden hierbei als regulative Handlungsfelder einer machtbewussten Beratung identifiziert. Dabei werden sowohl kulturelle Unterschiede (z. B. westliche bzw. östliche Kulturen) wie auch relevante Genderaspekte (weibliche bzw. männliche Machtzugänge) erörtert.

Gleich mehrfach stellt Theresia Volk zudem aktuelle Bezüge her, indem sie die sich derzeit vollziehenden Paradigmenwechsel herausarbeitet. Mit der Aussage „Beziehung vor Sache“ stellt sie die Dominanz der Rationalität über Emotionalität in sozialen Systemen grundsätzlich in Frage. Aus Disziplinarmacht wird so Selbstoptimierung, aus Befehlen wird Selbstverpflichtung und aus Prozesssicherheit wird Handlungsspielraum. Letztlich werden für Theresia Volk die traditionellen Legitimationsprinzipien ,Wahrheit und Konsens“ durch „Chance und Option“ ins Wanken gebracht.

Weniger auf eine stringente Argumentation und Beweisführung bedacht, vielmehr über gegensätzliche Positionen immer wieder Spannungsfelder aufsuchend und den eigenen Gedanken folgend, wird dem Leser von Theresia Volk mehr als nur ein Gedankensprung zugemutet. Dabei sind 
die so entstehenden Zusammenhänge so vielfältig, dass ein hohes Maß von Aufmerksamkeit von den Lesern gefordert wird.

„Spielen, um zu gewinnen.“ liest sich insgesamt flüssig und kommt ohne allzu komplexe sprachliche Strukturen aus, auch wenn zahlreiche Aufzählungen und Parenthesen $a b$ und zu etwas den Fluss brechen und den Leser zu kurzem Anhalten nötigen. Theresia Volk formuliert ihre Gedanken nachvollziehbar, ohne hierdurch glatt zu wirken und an notwendiger Reibungsfläche zu verlieren. In wörtlicher Rede kommen auf diese Art die unterschiedlichen Positionen unmittelbar zu Wort. Dabei steht weniger eine wissenschaftliche Nachweisführung des Gesagten als ein inspirierender Gedankenfluss im Vordergrund. Theresia Volk schafft es so, unzählige Anknüpfpunkte für den Leser bereitzuhalten.

Theresia Volk stellt sich mit dem Thema Macht und Wirksamkeit und dem Entwurf machtbewusster Beratung der Aufgabe, in der Reihe „Beraten in der Arbeitswelt“ sowohl konzeptionelle Basics als auch aktuelle Trends, in komprimierter Form bereitzustellen. Sie richtet sich mit dem Titel „Spielen, um zu gewinnen.“ sowohl an Beratungspraktiker wie Forscher und Lehrende. Dafür liefert sie in plausiblen praxisrelevanten Überlegungen einen nachvollziehbaren kompakten Überblick über relevante Faktoren von Machtkompetenz. Der Ansatz der machtbewussten Beratung stellt durch konkrete Hinweise und anwendbare Techniken einen hohen praktischen Nutzen für Berater und Ausbildende bereit. Forscher und Lehrende erhalten zahlreiche Denkanstöße, die nach weitergehender Betrachtung und wissenschaftlicher Aufarbeitung verlangen.

Mit spürbarer Begeisterung wie erkennbarer fachlicher Kompetenz für das Thema erfüllt Theresia Volk sowohl ihr Versprechen, den ,weiten Raum und Rahmen von Macht auszuleuchten", wie auch ihre eigene umfangreiche Erfahrung aus der Beratungspraxis in anwendungstauglicher Form bereitzustellen. Ersteres durch eine facettenreiche und von ihr immer wieder kontrovers ausgedeutete Literatursichtung, die von Machiavelli über Lewin bis Lobo führt. Letzteres führt zu einer konsequenten Herleitung der machtbewussten Beratung. Das Buch verlangt geradezu danach, das eigene Verhältnis zu und den Umgang mit der Macht - auch als Berater - zu überdenken. „Es gilt ganz generell, bei sich selbst die Fallen, Verführbarkeiten und Motive furchtlos zu erkunden." Genau dies erreicht Theresia Volk mit ihrem Buch „Spielen, um zu gewinnen.“

Eine gute Buchwahl für Berater und Lehrende, weil „Spielen, um zu gewinnen.“ sowohl konzeptionelle Ansätze als auch höchst aktuelle Entwicklungen berücksichtigt und dabei voller unbeantworteter Fragen bleibt. Das regt eigene Reflexion an, gerade auch weil eine wissenschaftliche Nachweisführung vieler Aussagen unterbleibt. Diese sich eröffnenden Fragestellungen laden die eher wissenschaftlich orientierten Leser zu einer weiteren Bearbeitung ein.

„Spielen, um zu gewinnen.“ ist ein sorgfältiges geplantes Puzzle aus kontroversen Positionen und Gedanken über die Bedeutung und Entwicklung von Machtkompetenzen, von Anfang bis Ende fesselnd und kann überzeugen.

Funding Open Access funding enabled and organized by Projekt DEAL.

Open Access Dieser Artikel wird unter der Creative Commons Namensnennung 4.0 International Lizenz veröffentlicht, welche die Nutzung, Vervielfältigung, Bearbeitung, Verbreitung und Wiedergabe in jeglichem Medium und Format erlaubt, sofern Sie den/die ursprünglichen Autor(en) und die Quelle ordnungsgemäß nennen, einen Link zur Creative Commons Lizenz beifügen und angeben, ob Änderungen vorgenommen wurden.

Die in diesem Artikel enthaltenen Bilder und sonstiges Drittmaterial unterliegen ebenfalls der genannten Creative Commons Lizenz, sofern sich aus der Abbildungslegende nichts anderes ergibt. Sofern das betreffende Material nicht unter der genannten Creative Commons Lizenz steht und die betreffende Handlung nicht nach gesetzlichen Vorschriften erlaubt ist, ist für die oben aufgeführten Weiterverwendungen des Materials die Einwilligung des jeweiligen Rechteinhabers einzuholen.

Weitere Details zur Lizenz entnehmen Sie bitte der Lizenzinformation auf http://creativecommons.org/licenses/by/4.0/deed.de.

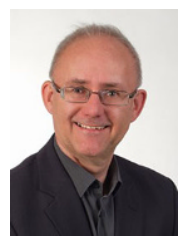

Martin Winand, geb.: 27.04.1065.

Diplomkaufmann

Bonn, Transformationsberater, Persönlichkeits- und Organisationsentwicklung.

Geschäftsführender Gesellschafter der focuslotsen UG (haftungsbeschränkt). Interessen- und Forschungsschwerpunk sind die psychologischen Faktoren und Dynamiken im Laufe von Transformationsprozessen von Führungssystemen aus der Perspektive mittlerer Führungsebenen. Trainer, Coach und Supervisor für Führungskräfte und Teams in agilen Arbeitskontexten und Entwicklungsprozesse. Projektmanager und agiler Praktiker seit 2003. 